\title{
A study on how to improve communication effectiveness of air traffic controllers
}

\author{
Yeong Heok Lee ${ }^{a}$, Jeong Dae Jeon ${ }^{b, *}$ and Gang Hyeon Lee ${ }^{c}$ \\ The School of Air Transport, Transportation \& Logistics, Korea Aerospace University, \\ Gyeonggi-Do, 412-791, Republic of Korea
}

\begin{abstract}
As the objective of air traffic control (ATC) services is to achieve flight safety and efficient aircraft operations, the role of air traffic controllers is vital in the aviation system as a result. In this paper, the controller's interactions were explored in terms of communications. The study was conducted through survey on the Republic of Korea Air Force (ROKAF) ATC controllers to identify any discrepancies in their viewpoints on the role of communication in their profession. In order to diminish differing points of view and ensure effective communication, the experienced controllers should commit to creating the desired work environment and every controller must keep in mind that mutual trust is a vital part of a successful organization. Instructions in accordance with the regulations, improvements in job satisfaction and self esteem, communication training, and individual endeavors fostering effective communication are all measures which will go a long way toward improving effective communications between the ATC specialists.
\end{abstract}

Keywords: Air Traffic Control, Flight Safety, Aircraft Operation, Aviation System, Controller Interaction, Communication Effectiveness

\footnotetext{
${ }^{a}$ Dr. Lee is a professor in the School of Air Transport, Transportation and Logistics at Korea Aerospace University where he specializes in aviation industries and policies. He has published more than 60 articles and books in academic journals such as Transport Research Record and Journal of Air Transport Management.

bJeon, Jeong Dae is a Ph. D candidate, Graduate School, Korea Aerospace University. As a military officer in ROKAF, he has been working in ATC fields for approximately 13 years.

${ }^{*}$ Corresponding author. E-mail: cometjeon@naver.com, phone: 82-2-300-0369

' Lee, Gang Hyeon is a Ph. D candidate, Graduate School, Korea Aerospace University. As a civil officer in KMOCT, he has been working in ATC fields for approximately 16 years.
} 


\section{I NTRODUCTION}

Since 1929 when the $1^{\text {st }}$ air traffic controller, Archie League, used colored flags to provide ATC services at St. Louis' Lambert Field airport, they have continuously evolved along with technical improvements in aircraft.

In accordance with Federal Aviation Administration Order (FAAO) 7110.65 'Air Traffic Control' and ICAO Doc 4444 'Rules of the Air and Air Traffic Services', ATC services are provided to prevent aircraft collisions and manage air traffic efficiently (FAA, 2000; ICAO, 1996). In other words, they are supposed to improve efficiency of air traffic management by preventing aircraft collisions and reducing unnecessary delays.

In reviewing commercial aircraft accident statistics issued by Boeing in 2001, approximately $70 \%$ of all commercial aircraft accidents occurred during take-off/cruising climb and final approach/landing stages, whereas accidents involving airport and ATC comprised only $3 \%$ of total accidents (Boeing, 2001). Human error, rather than technical failure, now represents the greatest threat to system reliability and safety in socio-technical systems such as aviation. The ratio of accidents caused by human error increased from $10 \%$ in the 1960 s to $80 \%$ in the 1990s. Its main reason was complexity of the new systems which improved reliability in technical components but increased the potential for human error by designers, decision makers, maintenance personnel, and so on (Airservices, 1996). The following statement illustrates how prone humans are to making mistakes: "To err is human and unfortunately, to err repeatedly is also human" (FAA, 1999).

An ATC specialist provides ATC services as a member of a team which is composed of pilots, other controllers, maintenance technicians and managers (D'Arcy and Pamela, 2001). Ineffective communications among the players adversely affect the individual controller's performance as well as team performance. As Heine Caesar insisted at Flight Safety Foundation Conference in 1990, communications should be trained in a community where order and obedience are a part of life (McCreary et al., 1998). The matter of communications should not be simply overlooked. As the performance model of flight crew, which was 
developed by 'Helmreich and Foushee' to optimize individual and team performance, shows, all inputs and components affecting outputs regarding aviation activities should be optimized. In this paper, by reviewing the interactions of ROKAF military ATC controllers in communication through surveys, we will determine the existence of any discrepancies in communication and the impediments to effective communication. Based upon the results, measures for more effective communication will be suggested to reduce verified shortfalls.

Accordingly, the related literature is reviewed to describe general ATC services. They explain what the team tasks in aviation and human factors are, how the human factors are applied in aviation, which communication process is involved in a human factors study, what relationships of power are formed in a small team consisting of personnel from the cockpit and the ATC facility, and what communications are like under stress. In order to identify the potential problems of communication between ATC controllers, empirical verifications will be implemented through survey data which incorporates data on information requests for assistance, comments on other's mistakes, personal preferences, and mutual trusts. Based on the data collected, statistical analysis including descriptive analysis, crosstabs and independent samples T-test will be conducted.

\section{LITERATURE REVIEW}

\subsection{ATC SERVICERS AND TEAMWORK I N ATC SYSTEMS}

ATC services are provided to prevent aircrafts from colliding each other and obstacles in the air and on the ground, and thus to maintain orderly, efficient and expeditious flow of air traffic. ATC services are divided into Aerodrome Control, Approach Control and Area Control Services in accordance with ICAO Annex 11 and Document 4444.

Figure 1 illustrates the flight flows of an aircraft and the ATC services provided for each flight stage by different ATC agencies. Aerodrome control services include issuing clearances for aircraft taking-off and landing, managing movement of aircraft and vehicles in the maneuvering area and delivering en route flight clearances to departing aircraft. Approach control facilities provide vectors for aircraft to enter into airways and to permit aircraft 
approaches to the airport. The area control center deals with the en route part of flights by assigning airways and altitudes to enable safe and expeditious flights, maintaining proper spacing between aircrafts, and providing information, such as NAVAIDs status, weather and airport status.

Figure 1: flight flows of an aircraft

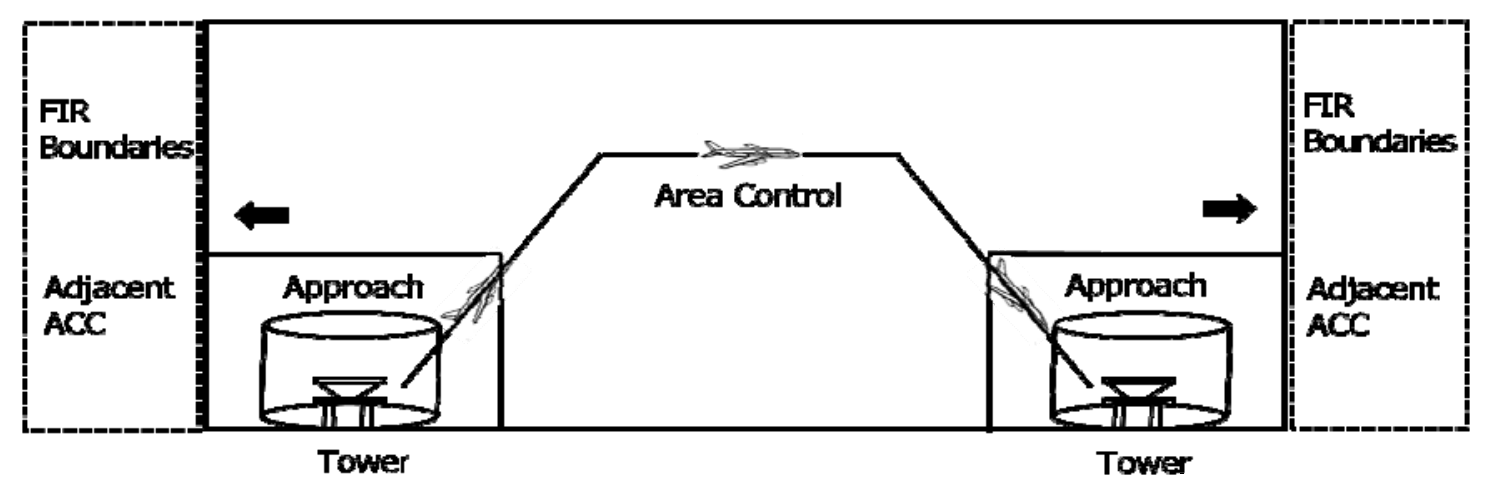

Due to ever increasing air traffic demands, it is expected that the capacity of the current ATC system will be saturated shortly (Air Transport Association, 1999). To cope with this capacity saturation in an effective and safe manner, ATC structures should see improvements with automation, satellite navigational systems and the application of data links, etc. But the options provided with these technical improvements should account for compatibilities with safety, efficiency, cost-effectiveness and human capabilities and limitations (Wickens et al., 1997). Safety should never be overlooked and any modifications or installations to the current systems must enhance flight safety.

Teamwork between ATC controllers is very important for safe and efficient air traffic management, because ATC has various team configurations and includes many actors, such as additional controllers who share functions in the same sector, supervisors and instructors, etc. Figure 2, which has been adapted from a performance model of flight crew, developed by 'Helmreich and Foushee', shows inputs, functions and outcomes in ATC. It shows how inputs, such as training, work attitudes and team composition, affect the functions and outcomes of an individual as well as a team, and how the outcomes influence the inputs and processing factors through proper feedback (Helmreich and Foushee', 1998). 
Figure 2: team performance model in ATC

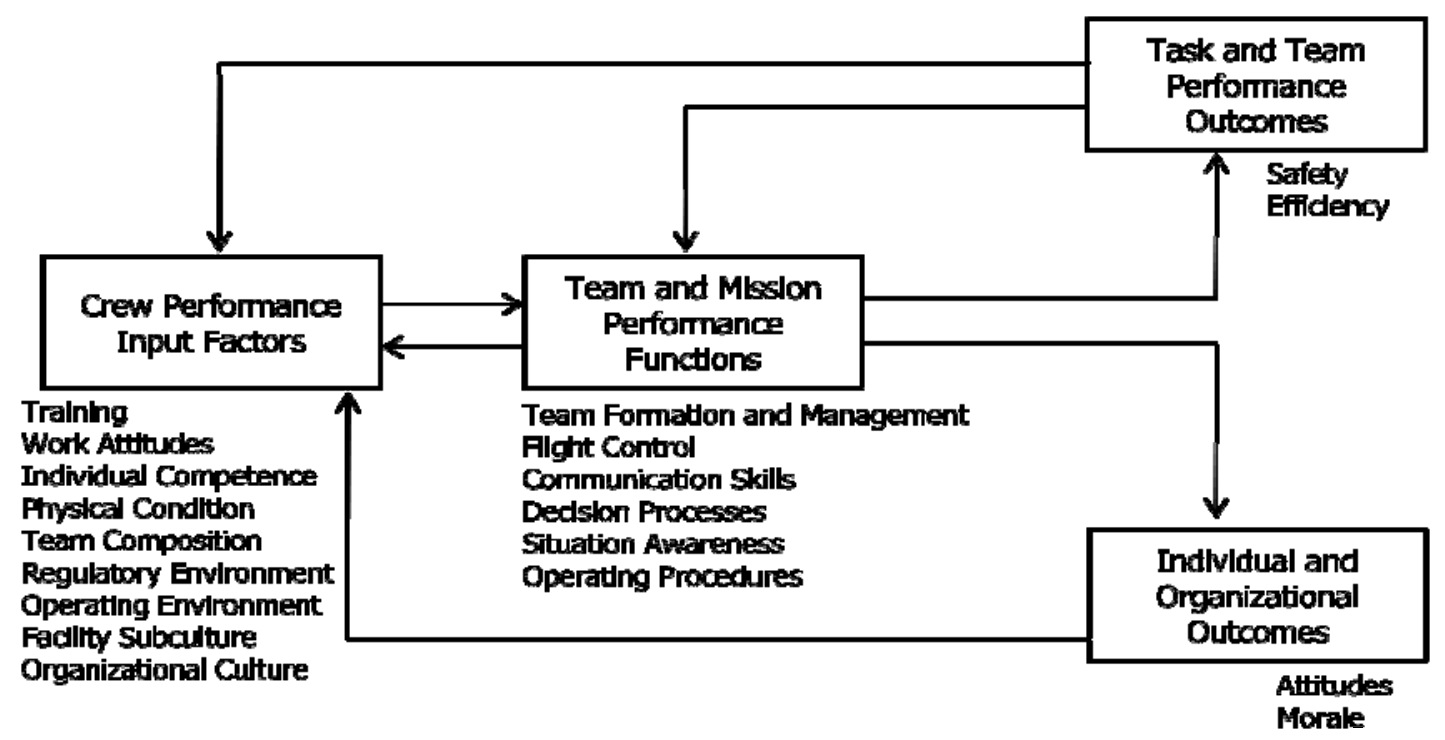

\subsection{HUMAN FACTORS IN ATC}

Currently, various definitions have been employed in attempts to describe the subject matter of human factors and one such definition can be as follows:

"Human factors may be defined as the technology concerned to optimize the relationships between people and their activities by the systematic application of the human sciences, integrated within the framework of system engineering." (Wiener and David, 1988)

In other words, understanding human factors is to understand human nature, such as the body as an organism, physiology, psychology, abilities and limitations, and to optimize human interactions with the surrounding environment for efficiency and safety from the related activities. Because human factors include all aspects of ergonomics, along with the events happening around humans, the extent and depth of this area is very broad and highly specialized. The SHEL model developed by Frank H. Hawkins in 1975 became the theoretical background for the human factors study in aviation by supporting the causes revealed in aircraft accident investigations (Korea Civil Aviation Development Association, 1996). Since many factors surrounding the centered human relate directly to aircraft operations, all the 
relevant factors inclusive of operators, equipment and work environments should be optimized in order to guarantee task efficiency and safety.

Humans and interactions between humans and systems are at the center of the human factors study in ATC, since humans influence systems in terms of safety, efficiency, capacity and treatment of uncertain conditions and the systems have an impact on human roles, functions, job satisfaction, health and morale, etc.

As an example of unwanted conditions between the centered human and the components in the SHEL model, we will see what can happen at the communication domain between controllers. A late decision to split a sector or the fear of 'losing face' in front of other controllers may cause undesired decision making so as to induce incident prone situations (EUROCONTROL, 1998).

In order to avoid these situations, ATC controllers must understand that ATC services are a team task and consider other controllers as available resources from whom they can get valuable information. It is strongly recommended to encourage controllers to interact actively in a systematic way (FAA, 1999).

\subsection{COMMUNI CATI ON I N A SMALL GROUP}

What is communication? Due to the broad range of communications, the general definitions of communication are too one-sided, complex or fractional. One way of defining communication is to explain the process associated with it. The reason for using the term 'process' for communication is that it is a progressing event (EUROCONTROL, 1997). Daily communications are usually composed of writing (word processing: 12\%), reading (letters, memos, etc.: $13 \%)$, speaking (30\%) and listening (45\%) (Parson, 1982).

Communication starts with an idea, a thought or an emotion and the sender translates the idea into symbols and sends a message to a receiver who decodes the message into information. As shown in figure 3, communication looks like a very simple process, but the receiver does not always understand your intentions and does not process your communications in the way you 
are expecting. Therefore, proper feedback is required to ensure the exact receipt of information as intended (EUROCONTROL, 1997).

Human communication takes place at two levels (Watzlawick et al., 1985). At level one, called the 'rational level', information is exchanged using words. Meanwhile, more meaning is embedded at level two, called the 'emotional level', where a general understanding of the situation is reached.

Figure 3: communication process

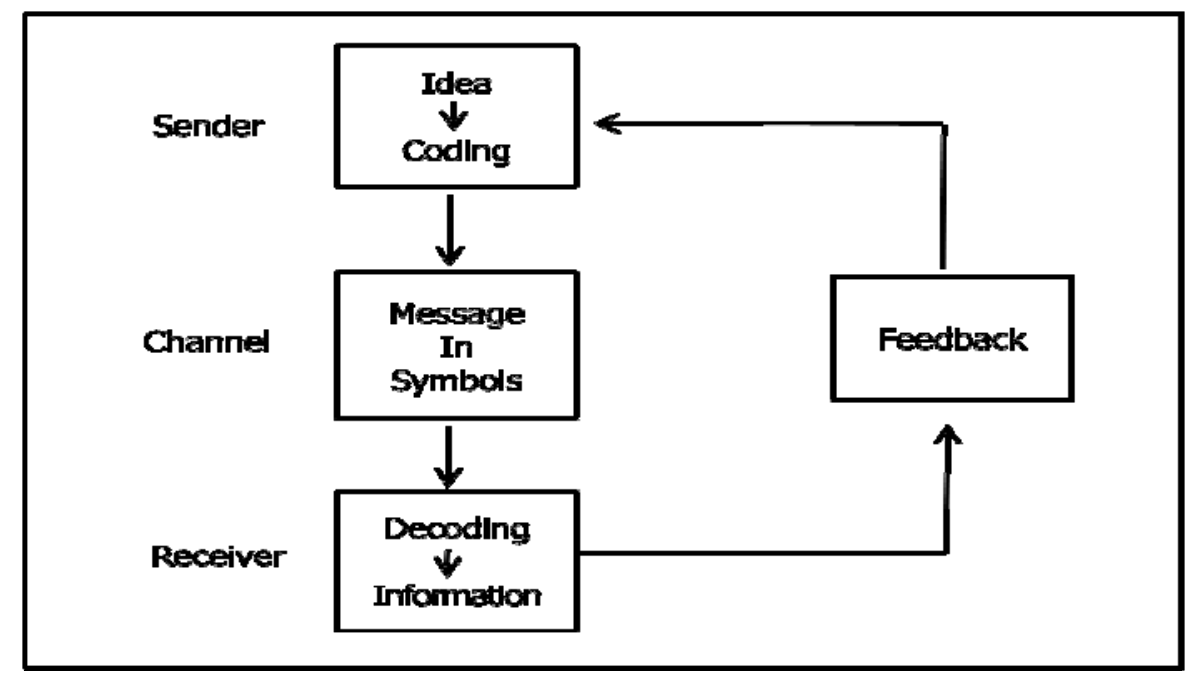

Each person possesses certain characteristics or properties which carry value for other people, and naturally, the greater number of highly valued properties a group member has, the more potential influence the person may have over the group. Five bases, namely reward, coerciveness, legitimacy, expertise and reference are suggested as power exercises to others (French and Bertram, 1968). As for military ATC facilities, military ranks form natural relationships for the controllers and thus the appropriate use of organic power is important for successful ATC operations.

For the groups, such as cockpits or ATC facilities which are prone to stress or crisis and depend highly on machinery, 't Hart et al conducted a survey of organizational behavior studies on crisis decision making and found three different communication patterns ('t Hart et al, 1993): 
- Instead of the group's normal decision making, the patterns of decision making become highly centralized.

- Because the decision maker should concentrate on the crisis situation, criticism, dissent and mutual recrimination must literally wait until the crisis is over.

- Inexperienced participants are often shut out of the centralized decision making process.

One thing we have to note is that while appropriate and legitimate decisions made by superiors in a crisis situation enables a swift and appropriate handling of problems, the exclusion of others with simple coercive power or dependence on prior experiences may result in shortfalls to aviation safety.

Each individual has various communication channels and the more channels a person has, the better he can communicate with others. Inevitably, there is always a leader in an ATC team and because the leader forms the culture of the team and influences the work environment and performance of other controllers, he should never neglect to make desirable work conditions for the team.

Successful communication relies on understanding communication processes and the methods for sending and receiving messages effectively and thus all ATC members must make other controllers understand clearly the messages they are sending and minimize mistakes the receivers may have, if any, through appropriate feedback. Leadership and communication style of superiors have a great impact on successful communication in a group. Although all members should try to have successful communication, superiors must actively lead others to form desirable organizational cultures and work environments, because subordinates usually have limited powers of influence on others. For superiors, the following tips will be helpful for improving interpersonal communication (EUROCONTROL, 1997):

- In selecting and treating the contents of a message, communication skill, attitude, knowledge, social status and culture of subordinates should be considered.

- To be the most effective, more than one communication channel has to be used.

- Superiors should try to make sure of the exact receipt of their messages from subordinates. 
- Superiors need to maintain proper feedback so as to reduce communication errors to determine the exactness of meaning and evaluate their influences.

- Superiors need to be reliable and objective and must have expertise.

\section{EMPI RI CAL STUDY}

In most industries, including the aviation industry, human factor studies and the application of them are becoming the main method for protecting people and property from disasters and for increasing productivity through efficiency of performance. In this study, a survey was conducted through ROKAF military ATC (tower and radar facilities) specialists to verify whether effective communication is taking place between controllers and whether there are any impediments to communication and to provide appropriate measures to ensure successful communication.

In the questionnaires, a five-Point Likert Scale is used to collect answers to the questions regarding degree of requesting help, commenting on other's mistakes and accepting attitudes, views on corrections and problems, considering other controller's situations, personal preferences, communication training and mutual trusts, and so on. There are two subjective questions in which controllers could freely express their opinion on obstacles to effective communication and the ways to improve communication between controllers.

The base for division of the two groups is a 10-year working career and careers of the superiors range from a minimum of 10 years to a maximum of 32 years and 6 months with standard deviations of 7 years and 4 months, while the subordinates have worked from 2 months to 9 years and 6 months with standard deviations of 2 years and 6 months. Data collection was conducted by mail. While 176 controllers participated in the study, only 105 responses (54 superiors and 51 subordinates) were determined to be useful samples with $59.6 \%$ response rate. SPSS 10.0 was used to analyze the data. In order to identify any discrepancies on communication viewpoints between controllers, descriptive analysis, crosstabs and T-test were conducted. 


\section{RESULTS}

The following table shows the items which have statistical importance at $p<.05$ in discrepancies in communication viewpoints between the two groups.

Table 1: Descriptive statistics and results of t-test

\begin{tabular}{|c|c|c|c|c|}
\hline Items & Groups & Means & SDs & T-Values \\
\hline \multirow{2}{*}{ Requesting for helps } & Subordinates & 3.27 & .90 & \multirow{2}{*}{-3.176} \\
\hline & Superiors & 3.83 & .91 & \\
\hline \multirow{2}{*}{$\begin{array}{c}\text { Commenting on other's } \\
\text { mistakes }\end{array}$} & Subordinates & 3.12 & .71 & \multirow{2}{*}{-2.151} \\
\hline & Superiors & 3.48 & 1.00 & \\
\hline \multirow{2}{*}{$\begin{array}{l}\text { Views on corrections } \\
\text { and problems }\end{array}$} & Subordinates & 3.16 & .70 & \multirow{2}{*}{-2.211} \\
\hline & Superiors & 3.48 & .79 & \\
\hline \multirow{2}{*}{ Considering others situations } & Subordinates & 2.88 & .74 & \multirow{2}{*}{-2.102} \\
\hline & Superiors & 3.22 & .90 & \\
\hline \multirow{2}{*}{$\begin{array}{l}\text { I nfluences of personal } \\
\text { relationships, such } \\
\text { as regional origins }\end{array}$} & Subordinates & 3.49 & .70 & \multirow{2}{*}{-2.719} \\
\hline & Superiors & 3.89 & .79 & \\
\hline \multirow{2}{*}{ Personal dislike of others } & Subordinates & 2.94 & .81 & \multirow{2}{*}{-2.144} \\
\hline & Superiors & 3.30 & .88 & \\
\hline \multirow{2}{*}{ Coping with problems } & Subordinates & 3.02 & .79 & \multirow{2}{*}{-2.187} \\
\hline & Superiors & 3.39 & .94 & \\
\hline
\end{tabular}

Regarding 'requesting for helps from other controllers when there are many aircrafts to deal with', discrepancies have been found between the two groups which show that superiors tend to ask for help from other controllers more easily than subordinates do, but one thing should be noted that this could happen as a result of the power that superiors have. As for commenting on other's mistakes, superiors addressed those matters more freely, while, to some degree, the two groups tended not to mention others' mistakes. This could mean that superiors are usually more responsible for ATC services. When corrections were required or 
problems were found in ATC operations, superiors tried more to resolve these challenges. Differences in considering other's situations existed and superiors tried to pay more attention to the subordinate's actions. School or regional ties was one factor in creating discrepancies of viewpoints between the two groups. Superiors responded to the question that it did not have a large impact on providing ATC services. Also, subordinates who are prone to be influenced easily by other controllers responded that there were some controllers whom they didn't like. When problems happened during ATC operations, superiors communicated more actively and positively with other controllers to resolve them. Even though mutual trust was a very important aspect for ATC operations, the results showed no statistical importance.

\subsection{IMPEDI MENTS TO COMMUNICATION AND ADVICE FOR COMMUNICATION EFFECTIVENESS}

Through two subjective questions, impediments to communication and methods to improve communications between controllers were collected and for these questions, $70 \%$ of superiors and $25 \%$ of subordinates responded.

Table 2: I mpediments to Communication

\begin{tabular}{c|c}
\hline I mpediments & Number of responses \\
\hline Unconditional Obedience & 17 \\
\hline Lack of Mutual Trusts & 6 \\
\hline Generation Gaps & 5 \\
\hline I llegitimate Orders & 5 \\
\hline Lack of Places and Time for Communication & 4 \\
\hline Selfishness & 4 \\
\hline Differences in Personalities & 3 \\
\hline Lack of Communication Skills & \\
\hline
\end{tabular}


As shown in Table 2, the main impediment to communication was unconditional obedience caused by military ranking structures. Lack of mutual trusts, generation gaps and illegitimate orders were included in this category. One thing that needed attention was the controllers' lack of communication skills, which required communication training to guarantee effective communication.

Table 3: Advices for Effective Communication

\begin{tabular}{c|c}
\hline Advices & Number of responses \\
\hline Mutual Trusts & 15 \\
\hline $\begin{array}{c}\text { Forming of Interrelationships through } \\
\text { Group Activities }\end{array}$ & 10 \\
\hline Considering Other's Viewpoints & 8 \\
\hline $\begin{array}{c}\text { Providing ATC I AW Regulations } \\
\text { Increasing J ob Satisfaction }\end{array}$ & 5 \\
\hline $\begin{array}{c}\text { Inducing the Feeling of } \\
\text { Accomplishment }\end{array}$ & 3 \\
\hline $\begin{array}{c}\text { Active Efforts of Superiors } \\
\text { (n) }\end{array}$ & 2 \\
\hline
\end{tabular}

Table 3 presents the advices for effective communication. It was found that effective communication depended on mutual trust and proper group activities provided a means for members to maintain favorable relationships. Maintaining consistency through the application of appropriate regulations improved communication effectiveness. It was also apparent that job satisfaction and accomplishment were important factors and in improving the communication process, superiors should make every effort to maximize both of these factors for their subordinates. Reviewing the responses by subordinates shows that as effort was required by superiors, the passive postures of subordinates caused by ranking structures could be guessed at.

\section{CONCLUSIONS}


In accordance with the results of this study which pursued to find any discrepancies on the communication viewpoints of military ATC controllers, superiors tended to address more freely than subordinates in requesting for helps and commenting on other's mistakes and dealt with corrections and problems more easily. Also, there was a difference in considering others' situations and personal preference influenced subordinates a lot.

Although the two groups did not have a specific plan for communication improvements, superiors faced little difficulty in initiating communication to cope with any problems they were facing. No statistical differences were found for mutual trust but superiors stated that they tried to communicate with others frankly.

As for impediments to effective communication, unconditional obedience caused by military ranking structures, lack of mutual trusts, generation gaps and lack of communication skills were identified as the main causes of miscommunication. To overcome these problems, mutual trusts, the forming of sound interrelationships through group activities, providing ATC IAW regulations and active efforts of superiors are required. But as mentioned above, ensuring effective communication depends upon understanding of the communication process by each individual and the active involvement of superiors to form sound structural cultures.

\section{LIMITATIONS AND RECOMMENDATIONS}

Because most studies of human factors in aviation have been related to pilots in the cockpit, it was difficult to collect any accident data for accidents caused by ATC controller communication problems. As human factors study for ATC has generally been conducted individually, identification of interrelationships between communication and performance was not convenient.

Because this study was based on a survey conducted through the postal service, it was not possible to get rid of the participant's errors in interpreting the questionnaire, which didn't permit refined analysis. 
It is recommended that based on the results of this study, in-depth studies should be conducted to find any relationships between communication and ATC controller's personal reliability, training and work attitudes, and so forth. Also structural and organized analysis is required for ATC team functions.

\section{References}

- Airservices (1996), Air Traffic Services: Human Factors Guide, Australia, pp.4-40.

- Air Transport Association (1999), Approaching Gridlock: Air Traffic Control Delays.

- Boeing (2001), Statistical Summary of Commercial Jet Airplane Accidents, pp.19-21.

- D'Arcy, J ean-Francois and Pamela S. Della Rocco (2001), Air Traffic Control Specialist Decision Making and Strategic Planning: A Field Survey.

- EUROCONTROL (1997), Human Factors Module: Interpersonal Communication, pp.58.

- EUROCONTROL (1998), Proceedings of the Second EUROCONTROL Human Factors Workshop: Teamwork in Air Traffic Services, pp.30-35.

- FAA (1999), Human Factors for Air Traffic Control Specialists: A User's Manual for Your Brain, pp.14-24.

- FAA, FAAO 7110.65M (2000), Air Traffic Control.

- Foushee, H. C. and Robert L. Helmreich (1998), Group interaction and flight crew performance, in Weiner, E. L. and Nagel, D. C., Human Factors in Aviation, pp.189-227.

- French, J. \& Bertram H. Raven (1968), In A. Zandler (Ed.) The base of social power in group dynamics: Research and theory (Ch. 20). New York: Harper \& Row.

- ICAO, Doc 4444-RAC/501 (1996), Rules of the Air and Air Traffic Control.

- Korea Civil Aviation Development Association (1996), Human Factors in Aviation, pp.15-19.

- McCreary, J., Michael Pollard, Kenneth Stevenson and Marc B. Wilson (1998), 'Human Factors: Tenerife Revisited'. Journal of Air Transportation World Wide, Vol. 3, No. 1, pp.23-31.

- Parson, G. (1982), Basics of Communication: The Communication Process.

- 't Hart, P., U. Rosenthal, \& A. Kouzmin (1993), Crisis decision making: The centralization thesis revisited. Administration \& Society, vol. 25, No 1. 
- Watzlawick, P. et al. (1985), Pragmatics of human communication. A study of Interactional Patterns, Pathologies, and Paradoxes. Wien: Huber.

- Wickens, Christopher D., Anne S. Mavor and James P. McGee (1997), Flight to the Future: Human Factors in Air Traffic Control. National Academy Press, Washington D.C.

- Wiener, Earl L. and David C. Nagel, (1988) Human Factors in Aviation, Academy Press, pp.7-10. 